\title{
Study on the Relationship between 5-HttLPR Gene and BDNF Gene Polymorphism and Post-Traumatic Stress Disorder in Li and Han Nationality of Hainan Province
}

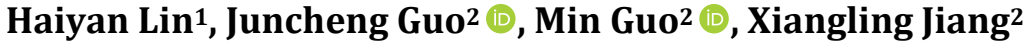 \\ ${ }^{1}$ The Fourth People's Hospital of Haikou, Haikou, China \\ ${ }^{2}$ Hainan Provincial People's Hospital, Haikou, China \\ Email: g2002m@163.com
}

How to cite this paper: Lin, H.Y., Guo, J.C., Guo, M. and Jiang, X.L. (2022) Study on the Relationship between 5-HttLPR Gene and BDNF Gene Polymorphism and PostTraumatic Stress Disorder in $\mathrm{Li}$ and Han Nationality of Hainan Province. Health, 14, 158-175.

https://doi.org/10.4236/health.2022.141012

Received: December 28, 2021

Accepted: January 24, 2022

Published: January 27, 2022

Copyright $\odot 2022$ by author(s) and Scientific Research Publishing Inc. This work is licensed under the Creative Commons Attribution International License (CC BY 4.0).

http://creativecommons.org/licenses/by/4.0/

\begin{abstract}
Objective: To investigate the correlation between 5-HTTLPR (5-and serotonin transporter linked polymer region) gene polymorphism and BDNF (brain derived neural factor) gene polymorphism and PTSD (post traumatic stress disorders) in $\mathrm{Li}$ and Han nationalities in Hainan Province. Methods: 167 Hainan Li PTSD patients, 141 Hainan Han PTSD patients and 158 healthy volunteers (control group) were investigated by ETI, caps, Toh, WCST, TMT and WAIS-RC. The polymorphisms of rs6265 locus of 5-HTTLPR and BDNF genes were detected by PCR (polymerase chain reaction) and page (polycylamide gel electrophoresis), and the correlation with PTSD was analyzed. Logistic regression analysis was used to analyze the influencing factors of PTSD. Results: The ETI score, total PTSD score and TMT time of Li PTSD patients were significantly higher than those of Han PTSD patients $(\mathrm{P}<0.01)$. The comprehension, picture filling, picture arrangement, operation IQ and total IQ of WAIS-RC were significantly lower than those of Han PTSD patients $(\mathrm{P}<$ 0.01 ); The numbers of errors, TMT and Toh in WCST were significantly lower than those in Han PTSD patients $(\mathrm{P}<0.01)$. There was no significant difference in the distribution of 5-HTTLPR genotype and allele between Li PTSD patients and control group (P > 0.05). SS genotype of 5-HTTLPR and $(\mathrm{GA}+\mathrm{AA})$ genotype of rs6265 locus may increase the risk of PTSD in Hainan Han population. AA and GA + AA genotypes at rs6265 locus may increase the risk of PTSD in Li population $(\mathrm{P}<0.05)$. Among Li PTSD patients, the ETI score, PTSD total score, TMT time, Toh planning time and execution time of AA genotype at rs6265 locus were significantly higher than those of GG genotype; the total scores of comprehension and operation IQ, and Toh
\end{abstract}


in WAIS-RC were significantly lower than those in GG genotype $(\mathrm{P}<0.05)$. Among Han PTSD patients, the ETI score, PTSD total score and TMT time of SS genotype of 5-HTTLPR were significantly higher than those of LL genotype, and the comprehension, arithmetic and block diagram in WAIS-RC were significantly lower than those of LL genotype; The ETI score, PTSD total score and TMT time of patients with (GA + AA) genotype at rs6265 locus were also significantly higher than those of patients with GG genotype. The comprehension and block diagram in WAIS-RC were significantly lower than those of patients with GG genotype. The number of WCST errors in patients with AA genotype was significantly higher than those of patients with GG genotype, and the operational IQ in WAIS-RC was significantly lower than those of patients with GG genotype $(\mathrm{P}<0.05)$. Conclusion: The LL genotype of 5-HTTLPR and the GG genotype of rs6265 locus are related to PTSD of Li and Han nationalities in Hainan, which are important protective factors for PTSD of Li and Han nationalities in Hainan.

\section{Keywords}

5-HTTLPR, BDNF, Gene Polymorphism, Post-Traumatic Stress Disorder, Li Nationality, Han Nationality, Frequency Distribution

\section{Preface}

Post-traumatic stress disorder (PTSD) is a debilitating anxiety disorder commonly seen in patients with emotional trauma [1]. Post traumatic stress disorder (PTSD) is a typical psychological stress disease. Post-traumatic stress disorder (PTSD) is a post-traumatic psychological disorder, which is a delayed and/or persistent anxiety response to unusually threatening or catastrophic events [2] [3]. People with PTSD often experience intrusive memories, avoidance and arousal symptoms, recurrent experiential memories, fear, and a range of persistent physical and behavioral sequelae [1] [4]. Women are more likely than men to develop PTSD, yet most people with PTSD do not receive or delay treatment. Currently, most treatments are administered after the onset of PTSD or even debilitating symptoms, and there is a lack of research focusing on the prevention, development and treatment of PTSD [5]. Lack of clinical subjects and short follow-up time are major obstacles to the prevention and development of PTSD. Due to its equal degree of heritability, the etiology of PTSD has been speculated to be related to complex gene-environment interactions [6] [7]. Some authors further speculate that individual differences in genetic susceptibility may play a role in the progression of PTSD, and that 5-HTTLPR polymorphisms and brainderived neurotrophic factor (BDNF) genotypes are viable approaches to finding effective PTSD treatments [8] [9]. Whether 5-HTTLPR and BDNF have the same correlation with PTSD patients of Li nationality and Han nationality in Hainan province has not been reported in China. Therefore, we hypothesized 
that genetic polymorphisms of 5-HTTLPR (VNTR) and BDNF (rs6265) were also associated with PTSD patients of $\mathrm{Li}$ and Han nationality. 5-HTTIPR is a form of $44 \mathrm{bp}$ insert/delete containing a two-cell, 16-element sequence [10]. There are two major functional variants of 5-HTTLPR (VNTR), namely the short (S, 14 repeat) allele and the long (L, 16 repeat) allele. The $S$ allele of 5-HTTLPR has been reported to increase the risk of PTSD in combat veterans [11]. Interestingly, a previous study found that 5-HTTLPR (VNTR) genotype may play an important role in the development and symptom severity of PTSD, and that 5-HTTLPR (VNTR) polymorphism may increase the risk of PTSD. The Sallele in 5-HTTLPR (VNTR) is associated with an increased risk for people with PTSD who lack social support, live in crime-ridden neighborhoods and have high unemployment or experience childhood adversity and traumatic events [12]. The association between 5-HTTLPR polymorphism and negative sexual events and major depression in Chinese population has been clarified, in which 5-HTTLPR polymorphism increases susceptibility to MDD in 20 - 29 year olds [13]. Neurotrophic factor (NFS) is related to the growth and survival of neurons during the development of the nervous system, and BDNF, as a type of NFS, is synthesized in primary sensory neurons and then transported to the primary afferent terminal of the dorsal horn of the central terminal spinal cord, and has been proved to be involved in the regulation of pain stimulation [14]. BDNF is a polypeptide growth factor, belonging to the neurotrophic factor family, which affects the development and treatment of various mental diseases, such as eating disorders, depression and anxiety disorders [8]. BDNF has been extensively studied and shown to be associated with synaptic plasticity processes that require long-term memory and learning, and has emerged as a novel approach to improve the efficacy of PTSD treatment [15] [16]. Mood disorders, especially PTSD, are the root of the influence of BDNF variation on psychiatric disorders, and THE BDNF genotype may serve as a biomarker to provide guidance for more personalized treatment [8]. There is evidence that BDNF gene variation may be a risk factor for Alzheimer's disease susceptibility in The Chinese population [17], as there are some ethnic differences in severity of PTSD symptoms among patients [18]. The aim of this study was to investigate the differences of 5-HTTLPR (VNTR) and BDNF (rs6265) polymorphisms and PTSD symptoms and other cognitive phenotypes in Li and Han populations in Hainan Province, China, in order to find the protective factors of PTSD.

\section{Data and Methods}

\subsection{The Research Object}

A total of 308 PTSD patients were enrolled in our hospital from October 2017 to July 2018 in Hainan Province, including 167 (54.2\%) from Li nationality and 141 (45.8\%) from Han nationality. 188 males and 120 females; The mean age was (45.2 \pm 5.6$)$ years. Inclusion criteria: 1$)$ PTSD was clearly diagnosed by 2 psychiatrists with intermediate professional title and above with clinical experience 
according to the unified diagnostic criteria (dsm-iv, PTSD) [19] [20]; 2) They have lived in Hainan for more than 10 years and are of the same nationality for three consecutive generations or more and have no kinship with each other; 3 ) 30 - 60 years old; 4) Feel good about your body. Exclusion criteria: a) family history of mental illness; b) patients with wasting diseases such as tumors; c) Pregnant women. 158 healthy volunteers who did not experience traumatic events and underwent physical examination in the health examination center of our hospital from October 2017 to July 2018 were selected as the control group, including 91 males and 67 females. The mean age was $(45.3 \pm 5.1)$ years. This study has been approved and supervised by the Ethics Committee of our hospital. All subjects in this study have obtained informed consent and signed informed consent by themselves and their family members.

\subsection{Detection of PTSD Indicators}

The Clinician PTSD Scale (CAPS) measures the frequency and intensity of PTSD symptoms using a separate 5-point scale (0 to 4$)$. Frequency and intensity ratings can be added to form a 9-point $(0-8)$ severity scale for various symptoms. Thus, CAPS can indicate the severity of PTSD symptoms at interview diagnosis on a scale of 0 to 136 [21]. Essen Trauma Inventory (ETI): Tagay S is a self-assessment questionnaire compiled in 2007 to assess traumatic events and their resulting psychological disorders (ASD and PTSD). The original questionnaire, in German, was based on the dSM-IV diagnosis of PTSD on a one-to-one basis. ETI consists of 58 items, which are divided into 5 parts. Part 3 is selected for study. Part 3 has 23 questions about current post-traumatic symptoms, including intrusions (B criteria, 5 items), avoidance ( $\mathrm{C}$ criteria, 7 items), and increased irritability (D criteria, 5 items), as well as asking about dissociation symptoms associated with acute stress disorder (6 items). ETI uses a 4-point score, with 0 meaning none at all, 1 meaning few, 2 meaning often and 3 meaning very much, for a total score of 23 items. The higher the total score, the more serious the psychological trauma [22]. Tower of Hanoi, TOH [23]: TOH is one of the best planned tests reflecting executive function. It can test the ability of planned adjustment, and it is also related to spatial perception, working memory, cognitive elasticity and interference suppression. 3-block and 4-block mobile manual plates were used in this study. Subjects are asked to move the wood block from the starting shape to the target shape according to certain rules. A total of 12 tests are included. Tests 1 to 6 are made up of 3 blocks, and tests 7 to 12 are made up of 4 blocks with increasing difficulty. The rules are: only one block can be moved at a time; Big blocks cannot be placed on top of small blocks; Wooden blocks can only be placed on the hand or post. Each task has 6 operation opportunities, two consecutive success (moves within 20 times) before the next task, two consecutive failures will stop the test. The evaluation parameters were total score, number of completed tasks, average planning time and average execution time. The higher the total score, the better the performance; the 
more completed tasks, the better the performance; the shorter the average planning time and average execution time, the better the performance [24]. Wsiconsin card sorting test (WCST): Requires the matching of one of four sorting cards to a stimulus type, which is defined in terms of color (C) shape (S) and number $(\mathrm{N})$ into multiple dimensions, each of which defines the sorting rules. Through trial and error, participants had to make choices (right or wrong) after being given sequential items on a screen. After 10 consecutive correct collation changes, there are up to six attempts to export rules, providing five rule shifts (C-S$\mathrm{N}-\mathrm{C}-\mathrm{S}-\mathrm{N}$ ) in the following order, each rule implementation called "complete a category." In the testing process, the participants could not know the correct sorting principle and main sorting changes; until all 128 cards are sorted, whether or not the participant has completed all rule change tests. There are two types of errors that can occur during this process. One is persistent error, in which the participants keep answering in the wrong order. The other is a nonpersistent error (random error) [25]. Trail Making Test (TMT): Tests mental processing speed, attention and cognitive ordering, spatial perception, eye-hand coordination, and mental agility. The test consists of two parts, a test and color test. TMT is divided into two parts: Part A requires subjects to connect 25 numbers on the paper in sequence and record the completion time; Part B included 25 figures in square and circle figures. Subjects were required to arrange the two figures alternately when connecting numbers in sequence, and record the completion time. Evaluation of measurement indexes: The less time consuming and the less interference, the better the executive function reflected Wechsler Adult Intelligence Scale Revised China (WAIS-RC): It included IQ scores in three categories: full Scale IQ, verbal IQ, and Operational IQ. Verbal IQ consists of seven sub-tests: vocabulary, similarity, arithmetic, number span, information, comprehension, and alphanumeric ordering. Operational INTELLIGENCE consists of six sub-tests: mapping, number symbol coding, group design, matrix reasoning, picture arrangement, and symbol search. The raw scores for these tests are converted to grade ratios.

\subsection{Gene Polymorphism Detection}

After the epidemiological investigation and test scale evaluation, $5-10 \mathrm{ml}$ of fasting anterior cubital vein blood was taken from all subjects in the morning, and EDTA anticoagulation was sent to the central Laboratory of Hainan Provincial People's Hospital for cryopreserved at $-20^{\circ} \mathrm{C}$. Genomic DNA was extracted by centrifuge column method using whole blood genomic DNA extraction Kit (OMEGA, USA), and the concentration and purity tests met the requirements of PCR amplification. At present, the primers commonly used in the world (original primers) are generally used in 5-HTTLPR, and this sequence is also used in this study (Table 1). PCR primers for rs6265 site of BDNF gene were designed using Primer Premier5.0 Primer design software (Table 1), and the primers were synthesized by Shanghai Shenggong Bioengineering Technology Service Co., 
Table 1. PCR primer sequences.

\begin{tabular}{ccc}
\hline SNP & & Sequence \\
\hline \multirow{2}{*}{ 5-HTTLPR } & Forward: & 5'-GGCGTTGCCGCTCTGAATGC-3' \\
& Reverse: & 5'-GAGGGACTGAGCTGGACAACCAC-3' \\
\hline \multirow{2}{*}{$B D N F$ gene rs6265 } & Forward: & 5'-TTTCTCCCTACAGTTCCACCAG-3' \\
& Reverse: & 5'-CTCCAAAGGCACTTGACTACTG-3' \\
\hline
\end{tabular}

LTD. PCR amplification reaction system was $50 \mu \mathrm{L}$, containing genomic DNA 1 $\mu \mathrm{L}$, a pair of primers $1 \mu \mathrm{L}(10 \mu \mathrm{M})$, PFU enzyme $0.25 \mu \mathrm{L}(5 \mathrm{U} / \mu \mathrm{L}), 10 \times$ Buffer 5 $\mu \mathrm{L}\left(200 \mathrm{mM}\right.$ TrisHCl, $\mathrm{pH} 8.8 ; 100 \mathrm{~mm} \mathrm{KCl} ; 20 \mathrm{~mm} \mathrm{MgSO}$; $160 \mathrm{~mm}\left(\mathrm{NH}_{4}\right) 2$ s04; $1 \%$ Triton, $1 \mathrm{mg} / \mathrm{mL} \mathrm{BSA})$, dNTPs $1 \mu \mathrm{L}(10 \mathrm{mM})$, deionized water $40.75 \mu \mathrm{L}$. PCR amplification cycle parameters: $98^{\circ} \mathrm{C}$ pre-denaturation for $3 \mathrm{~min}, 95^{\circ} \mathrm{C}$ denaturation for $1 \mathrm{~min}, 60^{\circ} \mathrm{C}$ annealing for 45 seconds, $72^{\circ} \mathrm{C}$ extension for 55 seconds, a total of 35 cycles, then $72^{\circ} \mathrm{C}$ extension for $8 \mathrm{~min}$. PCR kit was purchased from Shanghai Jierui Bioengineering Co., LTD. PCR products were sent to Shanghai Sangong Bioengineering Technology Service Co., LTD for sequencing.

\subsection{Statistical Analysis}

Statistical analysis was performed using SPSS 21.0 statistical software (SPSS Inc., Chicago, IL, USA). The measurement data were expressed as mean \pm standard deviation, and the comparison between the two groups was performed by $\mathrm{T}$ test for homogeneity of variance, and Wilcoxon rank sum test for non-homogeneity of variance or non-normal distribution. The counting data were expressed by composition ratio or ratio and chi-square test was used. Gene loci Hardy-Weinberg equilibrium test was $\chi^{2}$ test. The relative risk of genotype was expressed by odd ratio (OR) and 95\% confidence interval (CI). Chi-square test was used for frequency distribution between the two groups. The influencing factors of PTSD were analyzed by Logistic regression. "P" is bilateral probability, and $\mathrm{P}<0.05$ is statistically significant difference.

\section{Results}

\subsection{The Results of General Data Comparison of Subjects}

Among $167 \mathrm{Li}$ nationality PTSD patients, 98 were male and 69 were female. Average age was ( $45.4 \pm 5.3$ ) years; there were 90 males and 51 females in 141 Han nationality patients with PTSD. Average age was ( $44.9 \pm 5.8)$ years; there were 91 males and 67 females in 158 control groups. The mean age was (45.3 \pm 5.1) years. There were no significant differences in average age, sex composition, marital status and education level among the three groups (ALL P > 0.05). The ETI score, TOTAL PTSD score, TMT time, TOH planning time and execution time, WCST error number of Li nationality PTSD patients and Han nationality PTSD patients were significantly higher than the control group, waIS-RC 
comprehension, similarity, block map, object composition and operation IQ and TOTAL TOH were significantly lower than the control group. The ETI score, TOTAL PTSD score and TMT time of Li nationality PTSD patients were significantly higher than those of Han nationality PTSD patients, and the COMPREHENSION and operational IQ in WAIS-RC were significantly lower than those of Han nationality PTSD patients, with statistical significance (all $\mathrm{P}<$ 0.05) (Table 2).

\subsection{Genotype Analysis of rs6265 Polymorphisms of 5-HTTLPR and BDNF Genes}

528 bp and 484 bp gene fragments were detected by PCR amplification. The amplified products were divided into SS type SL type and LL type genotypes, with differences between LS and LL/SS, and the target fragment was tandem repeat. Therefore, genotypes of PCR products can be identified from the gel map without enzyme digestion (Figure 1(a)). After the PCR amplification product of rs6265 site of BDNF gene was cut by Eco72 I enzyme, there were mutant homozygous genotype AA (206 bp), mutant heterozygous genotype GA (206 bp, 137 bp and $69 \mathrm{bp}$ ) and wild homozygous genotype GG (137 bp and $69 \mathrm{bp}$ ) (Figure $1(b))$.

\subsection{Genotypes and Alleles of rs6265 Locus of 5-HTTLPR and BDNF Genes}

Goodness of fit test was used for Hardy-Weinberg equilibrium test, and the results showed that the genotypic distribution of 5-HTTLPR and BDNF rs6265 locus was in line with Hardy-Weinberg equilibrium (all $\mathrm{P}>0.05$ ). The samples in this study were random and representative of the population. There was no significant difference in the distribution of 5-HTTLPR genotype and allele between Li PTSD patients and the control group (ALL P > 0.05). The distribution of 5-HTTLPR genotype and allele was significantly different between Han nationality PTSD patients and control group: compared with LL genotype, SS genotype may increase the risk of PTSD in Han nationality $(\mathrm{OR}=2.157,95 \% \mathrm{CI}=$ 1.098 - 4.237, $\mathrm{P}=0.024$ ); Compared with $\mathrm{L}$ allele, $\mathrm{S}$ allele may increase the risk of PTSD in Han population $(\mathrm{OR}=1.456,95 \% \mathrm{CI}=1.045-2.207, \mathrm{P}=0.026)$. Compared with the control group, the distribution of genotype and allele of rs6265 locus of BDNF gene in Li nationality PTSD patients and Han Nationality PTSD patients were significantly different: Compared with GG genotype, GA genotype may increase the risk of PTSD in Han population $(\mathrm{OR}=2.210,95 \% \mathrm{CI}=$ 1.134 - 3.657, $\mathrm{P}=0.002)$. However, AA genotype may increase the risk of PTSD in Li nationality $(\mathrm{OR}=2.262,95 \% \mathrm{CI}=1.262-5.446, \mathrm{P}=0.008)$ and Han nationality $(\mathrm{OR}=4.358,95 \% \mathrm{CI}=2.057-9.230, \mathrm{P}<0.001)$. Compared with $\mathrm{G}$ allele, an allele may increase the risk of PTSD in $\mathrm{Li}$ population $(\mathrm{OR}=1.594,95 \% \mathrm{CI}=$ $1.145-2.221, \mathrm{P}=0.006)$ and Han population $(\mathrm{OR}=2.157,95 \% \mathrm{CI}=1.534$ 3.302, $\mathrm{P}=0.006) \mathrm{P}<0.001)($ Table 3, Table 4). 
Table 2. Comparison of general data of subjects.

\begin{tabular}{|c|c|c|c|c|c|}
\hline Items & $\begin{array}{l}\text { control group } \\
(\mathrm{n}=158)\end{array}$ & $\begin{array}{l}\text { Li PTSD group } \\
\quad(\mathrm{n}=167)\end{array}$ & $\begin{array}{l}\text { Han PTSD group } \\
\qquad(\mathrm{n}=141)\end{array}$ & $\mathrm{F}$ & $\mathrm{P}$ \\
\hline Average age & $45.3 \pm 5.1$ & $45.4 \pm 5.3$ & $44.9 \pm 5.8$ & 0.359 & 0.69880 \\
\hline Gender (Male/female) & $91 / 67$ & $98 / 69$ & $90 / 51$ & & \\
\hline Marital Status & $111 / 47$ & $101 / 66$ & $87 / 54$ & & \\
\hline Education (years) & $10.9 \pm 2.6$ & $10.6 \pm 2.6$ & $10.6 \pm 2.5$ & 0.711 & 0.49150 \\
\hline \multicolumn{6}{|l|}{ ETI score } \\
\hline Broke into symptoms & $3.13 \pm 0.93$ & $5.98 \pm 2.45$ & $4.97 \pm 1.89$ & 95.493 & 0.00000 \\
\hline Withdrawal symptoms & $3.63 \pm 1.15$ & $6.27 \pm 1.99$ & $4.67 \pm 1.57$ & 110.136 & 0.00000 \\
\hline $\begin{array}{l}\text { Hypervigilance } \\
\text { symptom }\end{array}$ & $3.49 \pm 1.08$ & $5.20 \pm 2.09$ & $4.70 \pm 1.42$ & 48.262 & 0.00000 \\
\hline separation of symptoms & $2.53 \pm 0.90$ & $4.40 \pm 1.63$ & $3.46 \pm 1.08$ & 89.877 & 0.00000 \\
\hline PTSD Total score & $15.14 \pm 6.13$ & $23.84 \pm 8.25$ & $19.54 \pm 6.29$ & 62.572 & 0.00000 \\
\hline \multicolumn{6}{|l|}{ WAIS-RC } \\
\hline knowledge & $10.67 \pm 1.75$ & $10.23 \pm 2.98$ & $10.52 \pm 2.58$ & 1.306 & 0.27187 \\
\hline understanding & $11.78 \pm 2.10$ & $9.86 \pm 2.47$ & $10.74 \pm 2.16$ & 29.407 & 0.00000 \\
\hline The arithmetic & $10.30 \pm 2.19$ & $9.89 \pm 2.82$ & $9.99 \pm 2.32$ & 1.197 & 0.30297 \\
\hline similar & $11.08 \pm 1.75$ & $10.34 \pm 2.62$ & $10.43 \pm 2.52$ & 2.369 & 0.09470 \\
\hline Digit span & $11.15 \pm 2.08$ & $10.44 \pm 3.56$ & $10.80 \pm 2.95$ & 1.343 & 0.26204 \\
\hline vocabulary & $10.30 \pm 2.19$ & $10.10 \pm 2.64$ & $10.54 \pm 2.14$ & 1.152 & 0.31687 \\
\hline Digit symbol & $10.46 \pm 1.46$ & $10.11 \pm 2.63$ & $10.13 \pm 2.62$ & 1.767 & 0.17207 \\
\hline Picture fill & $9.02 \pm 1.08$ & $8.59 \pm 2.56$ & $8.80 \pm 2.23$ & 16.073 & 0.00000 \\
\hline Block diagram & $10.78 \pm 2.10$ & $9.57 \pm 2.15$ & $9.97 \pm 1.47$ & 1.581 & 0.20695 \\
\hline Images are & $10.11 \pm 2.82$ & $9.62 \pm 2.49$ & $9.78 \pm 2.18$ & 54.629 & 0.00000 \\
\hline Objects together & $11.98 \pm 1.79$ & $9.83 \pm 2.18$ & $10.10 \pm 1.98$ & 1.951 & 0.14334 \\
\hline Language IQ & $101.37 \pm 15.01$ & $98.76 \pm 10.92$ & $99.43 \pm 10.2$ & 0.711 & 0.49150 \\
\hline Operation IQ & $101.58 \pm 10.00$ & $95.89 \pm 10.48$ & $98.76 \pm 13.2$ & 10.404 & 0.00004 \\
\hline total intelligence quotient (IQ) & $102.80 \pm 10.64$ & $97.59 \pm 9.71$ & $99.16 \pm 9.42$ & 11.588 & 0.00001 \\
\hline \multicolumn{6}{|l|}{ WCST } \\
\hline Class number & $4.11 \pm 0.93$ & $3.90 \pm 0.92$ & $3.91 \pm 1.03$ & 2.406 & 0.09134 \\
\hline Wrong number & $13.44 \pm 4.32$ & $18.80 \pm 4.45$ & $19.04 \pm 4.25$ & 82.819 & 0.00000 \\
\hline Correct number & $32.16 \pm 6.20$ & $30.90 \pm 6.03$ & $31.18 \pm 4.27$ & 2.213 & 0.11057 \\
\hline Persistent error number & $4.70 \pm 2.13$ & $5.02 \pm 2.37$ & $4.88 \pm 2.52$ & 0.762 & 0.46743 \\
\hline Random error number & $8.25 \pm 3.50$ & $8.63 \pm 3.02$ & $8.38 \pm 2.64$ & 0.637 & 0.52935 \\
\hline \multicolumn{6}{|l|}{ TMT } \\
\hline A Elapsed time (seconds) & $44.01 \pm 9.54$ & $53.83 \pm 12.07$ & $51.16 \pm 10.5$ & 35.355 & 0.00000 \\
\hline B Elapsed time (seconds) & $65.65 \pm 10.13$ & $79.60 \pm 8.97$ & $75.79 \pm 9.00$ & 94.557 & 0.00000 \\
\hline \multicolumn{6}{|l|}{$\mathrm{TOH}$} \\
\hline Plan time & $5.81 \pm 2.21$ & $7.28 \pm 2.32$ & $7.15 \pm 2.03$ & 21.632 & 0.00000 \\
\hline The execution time & $18.03 \pm 6.05$ & $26.07 \pm 6.05$ & $24.93 \pm 6.96$ & 74.683 & 0.00000 \\
\hline Total score & $54.69 \pm 8.55$ & $46.13 \pm 9.57$ & $47.15 \pm 8.66$ & 43.084 & 0.00000 \\
\hline
\end{tabular}

Note: ETI: Essen trauma inventory; TOH: Tower of Hanoi; WCST: Wsiconsin card sorting test; TMT: trail making test; WAIS-RC: Wechsler Adult Intelligence Scale revised Chin. 
H. Y. Lin et al.

Table 3. Comparison of genotype and allele frequency distribution of 5-HTTIPR and BDNF rs6265 locus between LI PTSD patients and control group [N (\%)].

\begin{tabular}{|c|c|c|c|c|}
\hline SNP & control group $(\mathrm{n}=158)$ & Li PTSD group $(n=167)$ & $\mathrm{P}$ & OR $(95 \% \mathrm{CI})$ \\
\hline \multicolumn{5}{|l|}{ 5-HTTLPR } \\
\hline LL & $35(22.2)$ & $26(15.6)$ & Ref. & \\
\hline LS & $68(43.0)$ & $83(49.7)$ & 0.141 & $0.640(0.352-1.161)$ \\
\hline SS & $55(34.8)$ & $58(34.7)$ & 0.325 & $0.732(0.392-1.364)$ \\
\hline LS+SS & $123(77.8)$ & $141(84.4)$ & 0.129 & $0.648(0.369-1.137)$ \\
\hline $\mathrm{L}$ & $138(43.7)$ & $135(40.4)$ & Ref. & \\
\hline S & $178(56.3)$ & $199(59.6)$ & 0.401 & $0.875(0.641-1.195)$ \\
\hline \multicolumn{5}{|l|}{$r s 6265$} \\
\hline GG & $84(53.2)$ & $69(41.3)$ & Ref. & \\
\hline GA & $61(38.6)$ & $70(41.9)$ & 0.161 & $1.397(0.875-2.232)$ \\
\hline AA & $13(8.2)$ & $28(16.8)$ & 0.008 & $2.262(1.262-5.446)$ \\
\hline $\mathrm{GA}+\mathrm{AA}$ & $74(46.8)$ & $98(58.7)$ & 0.033 & $1.162(1.039-2.500)$ \\
\hline G & $229(72.5)$ & $208(62.2)$ & Ref. & \\
\hline $\mathrm{A}$ & $87(27.5)$ & $126(37.8)$ & 0.006 & $1.594(1.145-2.221)$ \\
\hline
\end{tabular}

Note: SNP: Single Nucleotide Polymorphisms; OR: Odds Ratio; 95\% CI: 95\% confidence intervals; Ref: Reference.

Table 4. Comparison of genotype and allele frequency distribution of 5-HTTlPR and BDNF rs6265 locus between PTSD patients and control group [N (\%)].

\begin{tabular}{|c|c|c|c|c|}
\hline SNP & $\begin{array}{l}\text { control group } \\
\quad(\mathrm{n}=158)\end{array}$ & $\begin{array}{l}\text { Han PTSD group } \\
\qquad(\mathrm{n}=141)\end{array}$ & $P$ & OR (95\% CI) \\
\hline \multicolumn{5}{|l|}{ 5-HTTLPR } \\
\hline LL & $35(22.2)$ & $18(12.8)$ & Ref. & \\
\hline LS & $68(43.0)$ & $62(43.9)$ & 0.089 & $1.773(0.912-3.446)$ \\
\hline SS & $55(34.8)$ & $61(43.3)$ & 0.024 & $2.157(1.098-4.237)$ \\
\hline $\mathrm{LS}+\mathrm{SS}$ & $123(77.8)$ & $123(87.2)$ & 0.004 & $1.944(1.045-3.619)$ \\
\hline $\mathrm{L}$ & $138(43.7)$ & $98(34.8)$ & Ref. & \\
\hline$S$ & $178(56.3)$ & $184(65.2)$ & 0.026 & $1.456(1.045-2.027)$ \\
\hline \multicolumn{5}{|l|}{$r s 6265$} \\
\hline GG & $84(53.2)$ & $43(30.5)$ & Ref. & \\
\hline GA & $61(38.6)$ & $69(48.9)$ & 0.002 & $2.210(1.134-3.657)$ \\
\hline AA & $13(8.2)$ & $29(20.6)$ & $<0.001$ & $4.358(2.057-9.230)$ \\
\hline $\mathrm{GA}+\mathrm{AA}$ & $74(46.8)$ & $98(69.5)$ & $<0.001$ & $2.587(1.608-4.163)$ \\
\hline G & $229(72.5)$ & $155(54.9)$ & Ref. & \\
\hline A & $87(27.5)$ & $87(45.1)$ & $<0.001$ & $2.157(1.534-3.032)$ \\
\hline
\end{tabular}

Note: SNP: Single Nucleotide Polymorphisms; OR: Odds Ratio; 95\% CI: 95\% confidence intervals; Ref: Reference. 


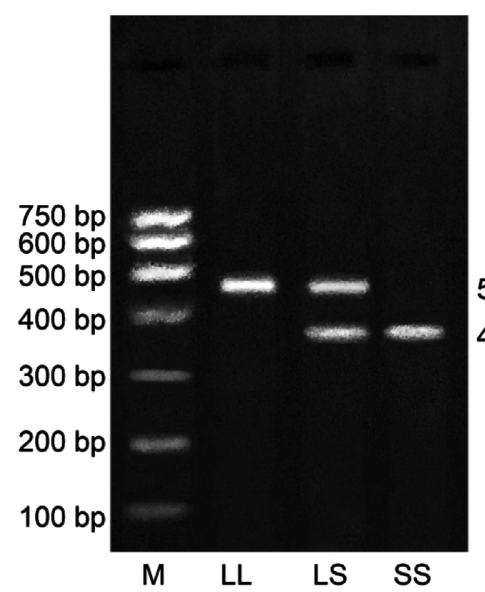

(a)

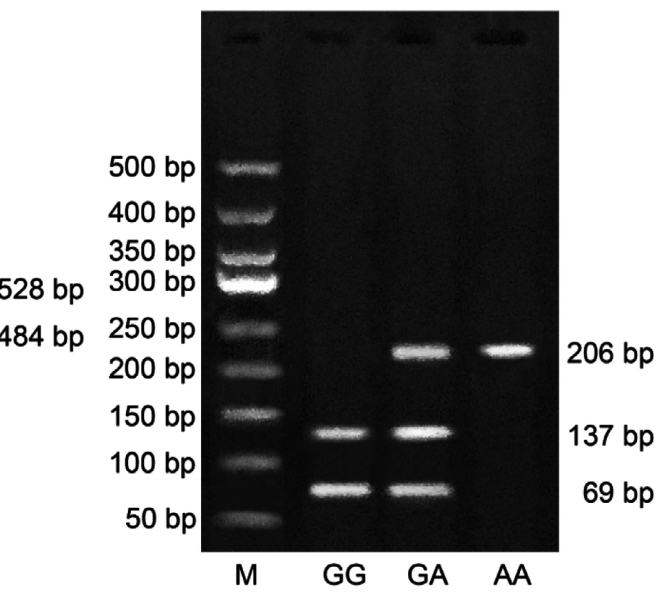

(b)

Figure 1. Sequencing results of PCR products of 5-HTTLPR (a) and (b) DNF rs6265 polymorphisms.

\subsection{Relationship between rs6265 Polymorphism of 5-HTTLPR and BDNF Gene and PTSD}

Among Li nationality PTSD patients, the ETI score, TOTAL PTSD score, TMT time, TOH planning time and execution time of AA genotype at rs6265 locus of BDNF gene were significantly higher than those of GG genotype. Comprehension and operational IQ, and TOH scores in WAIS-RC were significantly lower than those in GG genotype (all P < 0.05) (Table 5).

Among Han nationality PTSD patients: compared with LL genotype of 5HTTLPR, ETI score, PTSD total score and TMT time of SS genotype were significantly increased, and WAIS-RC comprehension and arithmetic, block diagram and operation IQ were significantly decreased (all $\mathrm{P}<0.05$ ). Compared with GG genotype of BDNF rs6265 locus, ETI score, PTSD total score and TMT time were significantly increased in (GA + AA) genotype, and comprehension and block map in WAIS-RC were significantly decreased. WCST errors in AA genotype were significantly higher than GG genotype. The operational IQ in WAIS-RC was significantly lower than that in GG genotype patients $($ all $\mathrm{P}<0.05)$ (Table 6).

\subsection{Logistic Regression Analysis}

The correlation between age, sex, marital status, education level, rs6265 polymorphism of 5-HTTLPR and BDNF gene and PTSD of Li and Han nationality was analyzed by binary Logistic regression analysis, with disease as the dependent variable. The results showed that: The LL genotype of 5-HTTLPR and THE GG genotype of rs6265 locus were significantly correlated with PTSD of Li and Han nationality, and were important protective factors for PTSD of Li and Han nationality (all $\mathrm{P}<0.05$ ). Age, sex, marital status and education level were not significantly correlated with PTSD of Li and Han nationality (all P > 0.05). As shown in Table 7. 
H. Y. Lin et al.

Table 5. 5-The relationship between HTTLPR and BDNF rs6265 polymorphism and PTSD in Li nationality.

\begin{tabular}{|c|c|c|c|c|c|c|}
\hline \multirow{2}{*}{ project } & \multicolumn{3}{|c|}{ 5-HTTLPR } & \multicolumn{3}{|c|}{ rs 6265} \\
\hline & $\mathrm{LL}(\mathrm{n}=26)$ & LS $(\mathrm{n}=83)$ & SS $(\mathrm{n}=58)$ & $\mathrm{GG}(\mathrm{n}=69)$ & $\mathrm{GA}(\mathrm{n}=70)$ & $\mathrm{AA}(\mathrm{n}=28)$ \\
\hline \multicolumn{7}{|l|}{ ETI score } \\
\hline Broke into symptoms & $6.46 \pm 3.05$ & $5.83 \pm 2.21$ & $5.98 \pm 2.49$ & $5.26 \pm 2.67$ & $6.20 \pm 2.36$ & $7.21 \pm 1.29^{\star}$ \\
\hline Withdrawal symptoms & $6.31 \pm 1.78$ & $6.15 \pm 1.90$ & $6.43 \pm 2.20$ & $5.67 \pm 2.29$ & $6.36 \pm 1.66$ & $7.46 \pm 1.35^{\star}$ \\
\hline $\begin{array}{l}\text { Hypervigilance } \\
\text { symptom }\end{array}$ & $4.89 \pm 2.37$ & $5.25 \pm 1.85$ & $5.23 \pm 2.32$ & $4.65 \pm 2.20$ & $5.39 \pm 1.94$ & $6.39 \pm 1.71^{\star}$ \\
\hline separation of symptoms & $4.42 \pm 1.45$ & $4.41 \pm 1.59$ & $4.38 \pm 1.80$ & $3.97 \pm 1.77$ & $4.51 \pm 1.42$ & $5.18 \pm 1.49^{\star}$ \\
\hline PTSD Total score & $23.38 \pm 10.25$ & $24.06 \pm 7.97$ & $23.74 \pm 7.80$ & $22.30 \pm 8.17$ & $24.04 \pm 8.79$ & $27.14 \pm 6.00^{\star}$ \\
\hline \multicolumn{7}{|l|}{ WAIS-RC } \\
\hline knowledge & $9.62 \pm 3.41$ & $10.61 \pm 2.88$ & $9.99 \pm 2.90$ & $10.36 \pm 2.96$ & $10.11 \pm 2.99$ & $10.18 \pm 3.13$ \\
\hline understanding & $9.42 \pm 2.37$ & $9.85 \pm 2.34$ & $10.05 \pm 2.70$ & $10.30 \pm 2.28$ & $9.87 \pm 2.69$ & $8.71 \pm 2.00^{*}$ \\
\hline The arithmetic & $9.46 \pm 2.76$ & $10.17 \pm 2.96$ & $9.67 \pm 2.65$ & $9.59 \pm 3.02$ & $10.17 \pm 2.66$ & $9.89 \pm 2.75$ \\
\hline similar & $10.54 \pm 2.50$ & $10.07 \pm 2.56$ & $10.62 \pm 2.82$ & $10.20 \pm 2.83$ & $10.51 \pm 2.26$ & $10.21 \pm 3.07$ \\
\hline Digit span & $10.73 \pm 3.46$ & $10.45 \pm 3.46$ & $10.28 \pm 3.79$ & $10.26 \pm 3.68$ & $10.13 \pm 3.38$ & $11.61 \pm 3.61$ \\
\hline vocabulary & $9.39 \pm 2.43$ & $10.16 \pm 2.59$ & $10.33 \pm 2.76$ & $10.13 \pm 2.58$ & $10.17 \pm 2.64$ & $9.82 \pm 2.80$ \\
\hline Digit symbol & $10.15 \pm 2.24$ & $10.20 \pm 2.75$ & $9.97 \pm 2.67$ & $10.13 \pm 2.57$ & $10.07 \pm 2.69$ & $10.18 \pm 2.74$ \\
\hline Picture fill & $8.62 \pm 2.93$ & $8.60 \pm 2.50$ & $8.57 \pm 2.51$ & $8.68 \pm 2.67$ & $8.50 \pm 2.56$ & $8.61 \pm 2.36$ \\
\hline Block diagram & $9.58 \pm 1.86$ & $9.64 \pm 2.22$ & $9.41 \pm 2.18$ & $9.54 \pm 2.09$ & $9.54 \pm 2.21$ & $9.61 \pm 2.18$ \\
\hline Images are & $10.04 \pm 2.84$ & $9.51 \pm 2.54$ & $9.60 \pm 2.26$ & $9.39 \pm 2.72$ & $9.99 \pm 2.05$ & $9.29 \pm 2.84$ \\
\hline Objects together & $9.19 \pm 2.02$ & $9.93 \pm 2.15$ & $9.98 \pm 2.28$ & $9.88 \pm 2.15$ & $9.90 \pm 2.16$ & $9.54 \pm 2.37$ \\
\hline Language IQ & $99.81 \pm 12.65$ & $98.45 \pm 9.84$ & $99.19 \pm 11.74$ & $98.55 \pm 11.56$ & $98.46 \pm 10.96$ & $100.0 \pm 9.36$ \\
\hline Operation IQ & $95.46 \pm 9.50$ & $96.86 \pm 10.33$ & $94.71 \pm 11.14$ & $99.17 \pm 9.77$ & $96.09 \pm 8.60$ & $87.32 \pm 11.95^{\star}$ \\
\hline $\begin{array}{l}\text { total intelligence } \\
\text { quotient (IQ) }\end{array}$ & $98.54 \pm 10.98$ & $97.51 \pm 9.56$ & $97.28 \pm 9.49$ & $98.32 \pm 9.34$ & $97.23 \pm 9.63$ & $96.68 \pm 10.99$ \\
\hline \multicolumn{7}{|l|}{ WCST } \\
\hline Class number & $4.08 \pm 0.98$ & $3.80 \pm 0.88$ & $3.97 \pm 0.95$ & $3.84 \pm 0.99$ & $3.89 \pm 0.83$ & $4.07 \pm 0.98$ \\
\hline Wrong number & $18.92 \pm 4.85$ & $18.72 \pm 4.08$ & $18.86 \pm 4.85$ & $18.46 \pm 4.81$ & $19.20 \pm 3.94$ & $18.64 \pm 4.81$ \\
\hline Correct number & $31.81 \pm 4.85$ & $30.78 \pm 6.61$ & $30.66 \pm 5.67$ & $30.83 \pm 6.28$ & $30.80 \pm 5.88$ & $31.32 \pm 5.94$ \\
\hline Persistent error number & $4.07 \pm 1.96$ & $5.23 \pm 2.47$ & $5.16 \pm 2.34$ & $5.23 \pm 2.47$ & $4.99 \pm 2.07$ & $4.61 \pm 2.83$ \\
\hline Random error number & $8.23 \pm 2.88$ & $8.90 \pm 3.00$ & $8.41 \pm 3.11$ & $8.61 \pm 3.18$ & $8.74 \pm 3.02$ & $8.39 \pm 2.67$ \\
\hline \multicolumn{7}{|l|}{ TMT } \\
\hline A Elapsed time (seconds) & $55.19 \pm 9.82$ & $53.35 \pm 12.65$ & $53.09 \pm 12.27$ & $49.54 \pm 10.47$ & $52.79 \pm 12.49$ & $66.11 \pm 5.98^{\star}$ \\
\hline B Elapsed time (seconds) & $81.23 \pm 7.98$ & $79.12 \pm 8.67$ & $79.57 \pm 9.83$ & $76.26 \pm 9.87$ & $79.17 \pm 5.14$ & $88.93 \pm 7.91^{\star}$ \\
\hline \multicolumn{7}{|l|}{$\mathrm{TOH}$} \\
\hline Plan time & $7.50 \pm 2.05$ & $6.95 \pm 2.27$ & $7.64 \pm 2.48$ & $7.00 \pm 2.22$ & $6.73 \pm 2.12$ & $9.32 \pm 1.96^{*}$ \\
\hline The execution time & $25.73 \pm 5.41$ & $26.48 \pm 5.99$ & $25.64 \pm 6.45$ & $24.22 \pm 6.55$ & $26.21 \pm 2.55$ & $30.29 \pm 8.56$ \\
\hline Total score & $46.73 \pm 8.08$ & $45.54 \pm 9.63$ & $46.71 \pm 10.18$ & $45.71 \pm 9.82$ & $43.07 \pm 8.47$ & $54.82 \pm 5.75^{\star}$ \\
\hline
\end{tabular}

Note: ETI: Essen Trauma Inventory; TOH: Tower of Hanoi; WCST: Wsiconsin Card sorting test; TMT: trail making test; Wais-rc: Wechsler Adult Intelligence Scale Revised Chin; *compared with LL genotype of 5-HTTlPR and GG genotype of rs6265 in LI PTSD patients, $\mathrm{P}<0.05$. 
Table 6. 5-The relationship between HTTLPR and BDNF rs6265 polymorphism and PTSD in Han Nationality.

\begin{tabular}{|c|c|c|c|c|c|c|}
\hline \multirow{2}{*}{ project } & \multicolumn{3}{|c|}{ 5-HTTLPR } & \multicolumn{3}{|c|}{$r s 6265$} \\
\hline & $\mathrm{LL}(\mathrm{n}=18)$ & $\operatorname{LS}(n=62)$ & $\operatorname{SS}(n=61)$ & GG $(n=43)$ & $\mathrm{GA}(\mathrm{n}=68)$ & $\mathrm{AA}(\mathrm{n}=29)$ \\
\hline \multicolumn{7}{|l|}{ ETI score } \\
\hline Broke into symptoms & $3.72 \pm 1.07$ & $4.79 \pm 2.15$ & $5.53 \pm 1.48^{\star}$ & $3.44 \pm 0.80$ & $5.80 \pm 1.64^{*}$ & $5.28 \pm 2.09^{*}$ \\
\hline Withdrawal symptoms & $3.66 \pm 1.33$ & $4.05 \pm 1.08$ & $5.59 \pm 1.59^{*}$ & $3.95 \pm 1.02$ & $4.88 \pm 1.38^{\star}$ & $5.21 \pm 2.23^{\star}$ \\
\hline $\begin{array}{l}\text { Hypervigilance } \\
\text { symptom }\end{array}$ & $3.67 \pm 1.28$ & $4.32 \pm 1.39$ & $5.44 \pm 1.06^{\star}$ & $3.37 \pm 0.90$ & $5.28 \pm 1.08^{\star}$ & $5.31 \pm 1.44^{*}$ \\
\hline separation of symptoms & $2.56 \pm 1.04$ & $3.15 \pm 0.96$ & $4.05 \pm 0.88^{\star}$ & $2.49 \pm 0.77$ & $3.80 \pm 0.68^{\star}$ & $4.08 \pm 1.12^{\star}$ \\
\hline PTSD Total score & $15.44 \pm 7.46$ & $17.69 \pm 4.78$ & $22.62 \pm 5.92^{\star}$ & $16.23 \pm 5.81$ & $20.62 \pm 4.58^{\star}$ & $21.86 \pm 8.42^{\star}$ \\
\hline \multicolumn{7}{|l|}{ WAIS-RC } \\
\hline knowledge & $10.44 \pm 2.50$ & $10.40 \pm 2.92$ & $10.67 \pm 2.26$ & $10.37 \pm 2.80$ & $10.61 \pm 2.56$ & $10.55 \pm 2.38$ \\
\hline understanding & $12.11 \pm 3.27$ & $12.08 \pm 0.87$ & $8.99 \pm 1.22^{\star}$ & $12.40 \pm 2.13$ & $10.86 \pm 1.18^{\star}$ & $8.03 \pm 1.12^{\star}$ \\
\hline The arithmetic & $11.56 \pm 2.68$ & $10.92 \pm 2.19$ & $8.57 \pm 1.41^{\star}$ & $11.12 \pm 2.52$ & $10.22 \pm 1.87$ & $7.76 \pm 1.30^{*}$ \\
\hline similar & $10.50 \pm 2.46$ & $10.10 \pm 2.86$ & $10.74 \pm 2.15$ & $10.19 \pm 2.61$ & $10.48 \pm 2.57$ & $10.66 \pm 2.33$ \\
\hline Digit span & $11.11 \pm 2.81$ & $10.84 \pm 2.93$ & $10.67 \pm 3.04$ & $11.19 \pm 2.97$ & $10.62 \pm 2.94$ & $10.66 \pm 3.02$ \\
\hline vocabulary & $10.78 \pm 1.99$ & $10.39 \pm 2.40$ & $10.62 \pm 1.92$ & $10.56 \pm 2.29$ & $10.55 \pm 2.13$ & $10.48 \pm 1.99$ \\
\hline Digit symbol & $10.50 \pm 2.75$ & $10.05 \pm 2.60$ & $10.11 \pm 2.63$ & $10.65 \pm 2.60$ & $9.65 \pm 2.77$ & $10.52 \pm 2.10$ \\
\hline Picture fill & $9.16 \pm 2.43$ & $8.74 \pm 2.20$ & $8.75 \pm 2.23$ & $9.23 \pm 2.25$ & $8.44 \pm 2.34$ & $9.03 \pm 1.82$ \\
\hline Block diagram & $11.06 \pm 1.11$ & $10.61 \pm 1.06$ & $9.00 \pm 1.34^{*}$ & $11.26 \pm 0.82$ & $9.55 \pm 0.95^{\star}$ & $9.07 \pm 1.94^{*}$ \\
\hline Images are & $10.17 \pm 2.09$ & $9.45 \pm 2.46$ & $10.00 \pm 1.88$ & $9.63 \pm 2.34$ & $9.87 \pm 2.18$ & $9.79 \pm 2.02$ \\
\hline Objects together & $9.72 \pm 1.60$ & $10.42 \pm 1.92$ & $9.88 \pm 2.13$ & $10.19 \pm 1.68$ & $10.22 \pm 2.11$ & $9.69 \pm 2.12$ \\
\hline Language IQ & $100.3 \pm 9.49$ & $98.68 \pm 11.58$ & $99.92 \pm 9.02$ & $99.70 \pm 10.09$ & $99.23 \pm 10.83$ & $99.48 \pm 9.26$ \\
\hline Operation IQ & $107.7 \pm 15.93$ & $102.1 \pm 11.76$ & $92.70 \pm 11.16^{\star}$ & $103.7 \pm 13.93$ & $98.71 \pm 10.25$ & $91.52 \pm 15.49^{*}$ \\
\hline $\begin{array}{l}\text { total intelligence quotient } \\
\text { (IQ) }\end{array}$ & $101.6 \pm 9.17$ & $98.40 \pm 10.50$ & $99.21 \pm 8.32$ & $99.79 \pm 9.72$ & $98.78 \pm 9.94$ & $99.14 \pm 7.89$ \\
\hline \multicolumn{7}{|l|}{ WCST } \\
\hline Class number & $4.06 \pm 1.00$ & $3.73 \pm 1.12$ & $4.05 \pm 0.92$ & $3.84 \pm 1.13$ & $3.93 \pm 0.99$ & $3.97 \pm 0.98$ \\
\hline Wrong number & $18.11 \pm 6.18$ & $18.21 \pm 1.90$ & $20.15 \pm 5.05$ & $17.63 \pm 4.29$ & $17.86 \pm 2.86$ & $23.93 \pm 3.41^{\star}$ \\
\hline Correct number & $32.22 \pm 3.75$ & $30.29 \pm 4.67$ & $31.79 \pm 3.85$ & $30.88 \pm 4.46$ & $31.19 \pm 4.22$ & $31.62 \pm 4.20$ \\
\hline Persistent error number & $4.89 \pm 3.18$ & $4.94 \pm 2.30$ & $4.80 \pm 2.61$ & $4.70 \pm 2.60$ & $5.12 \pm 2.50$ & $4.59 \pm 2.60$ \\
\hline Random error number & $7.50 \pm 2.96$ & $8.50 \pm 2.71$ & $8.51 \pm 2.47$ & $8.26 \pm 2.99$ & $8.42 \pm 2.40$ & $8.45 \pm 2.72$ \\
\hline \multicolumn{7}{|l|}{ TMT } \\
\hline A Elapsed time (seconds) & $48.89 \pm 15.90$ & $45.66 \pm 3.54$ & $57.43 \pm 10.18^{\star}$ & $45.02 \pm 10.71$ & $52.48 \pm 5.52^{\star}$ & $57.14 \pm 14.60^{*}$ \\
\hline B Elapsed time (seconds) & $71.50 \pm 7.46$ & $72.65 \pm 7.20$ & $80.26 \pm 9.18^{\star}$ & $70.91 \pm 9.45$ & $76.93 \pm 3.60^{*}$ & $80.34 \pm 13.34^{*}$ \\
\hline \multicolumn{7}{|l|}{$\mathrm{TOH}$} \\
\hline Plan time & $7.17 \pm 1.98$ & $7.00 \pm 2.32$ & $7.30 \pm 1.74$ & $7.05 \pm 2.21$ & $7.22 \pm 1.95$ & $7.14 \pm 2.00$ \\
\hline The execution time & $21.94 \pm 7.79$ & $24.79 \pm 7.08$ & $25.95 \pm 6.43$ & $23.72 \pm 7.87$ & $25.03 \pm 6.36$ & $27.48 \pm 6.19$ \\
\hline Total score & $47.44 \pm 8.56$ & $46.48 \pm 9.90$ & $47.74 \pm 7.33$ & $46.98 \pm 9.40$ & $47.32 \pm 8.46$ & $47.00 \pm 8.29$ \\
\hline
\end{tabular}

Note: ETI: Essen Trauma Inventory; TOH: Tower of Hanoi; WCST: Wsiconsin Card sorting test; TMT: trail making test; Wais-rc: Wechsler Adult Intelligence Scale Revised Chin; ${ }^{\star}$ compared with LL genotype of 5-HTTlPR and GG genotype of rs6265 in LI PTSD patients, $\mathrm{P}<0.05$. 
Table 7. Logistic regression analysis.

\begin{tabular}{|c|c|c|c|c|c|c|c|}
\hline \multirow{2}{*}{ Factor } & \multirow{2}{*}{ B } & \multirow{2}{*}{ S.E. } & \multirow{2}{*}{ Wald } & \multirow{2}{*}{ sig. } & \multirow{2}{*}{$\operatorname{Exp}(B)$} & \multicolumn{2}{|c|}{ 95\% CI for $\operatorname{Exp}(B)$} \\
\hline & & & & & & Lower & Upper \\
\hline \multicolumn{8}{|l|}{$\mathrm{Li}$} \\
\hline age & 0.010 & 0.022 & 0.022 & 0.649 & 1.010 & 0.967 & 1.056 \\
\hline gender & 0.481 & 0.276 & 0.276 & 0.082 & 1.618 & 0.942 & 2.780 \\
\hline Marital status & -0.337 & 0.291 & 0.291 & 0.247 & 0.714 & 0.404 & 1.262 \\
\hline Education Level & -0.050 & 0.044 & 0.044 & 0.257 & 0.951 & 0.872 & 1.037 \\
\hline 5-HTTLPR & -0.865 & 0.305 & 0.305 & 0.005 & 0.421 & 0.232 & 0.765 \\
\hline rs6265 & -0.659 & 0.265 & 0.265 & 0.013 & 0.517 & 0.308 & 0.870 \\
\hline \multicolumn{8}{|l|}{ Han } \\
\hline age & -0.009 & 0.023 & 0.157 & 0.692 & 0.991 & 0.948 & 1.036 \\
\hline gender & 0.515 & 0.281 & 3.368 & 0.066 & 1.674 & 0.966 & 2.901 \\
\hline Marital status & -0.239 & 0.293 & 0.663 & 0.416 & 0.788 & 0.444 & 1.399 \\
\hline Education Level & -0.051 & 0.048 & 1.112 & 0.292 & 0.950 & 0.865 & 1.045 \\
\hline 5-HTTLPR & -0.800 & 0.384 & 4.349 & 0.037 & 0.449 & 0.212 & 0.953 \\
\hline rs6265 & -0.973 & 0.306 & 10.095 & 0.001 & 0.378 & 0.207 & 0.689 \\
\hline
\end{tabular}

Note: B: partial regression coefficient; S.E: (Standard Error; Sig: significance; OR: Odds ratio; CI: confidence interval).

\section{Discussion}

PTSD is a complex mental disorder that can occur during traumatic events, such as car accidents, rape, combat exposure or natural disasters [26]. Post-traumatic stress disorder imposes a serious health, social and economic burden on individuals and society as a whole [27]. It has been reported that 5-HTTLPR polymorphism and BDNF genotype play an important role in the occurrence and treatment of PTSD [4] [16] [28]. To investigate the effect of 5-HTTLPR (VNTR) and BDNF (rs6265) gene polymorphisms on PTSD, our present study included $\mathrm{Li}$ and Han PTSD patients from Hainan Province. We found that the ETI score, TOTAL PTSD score, TMT time, TOH planning time and execution time, and WCST errors of the two groups of PTSD patients were higher than those of the control group. Compared with the control group, the WAIS-RC similarity, chunking pattern, understanding, object assembly and operation IQ of PTSD patients were decreased. Suggesting that PTSD affects people's general ability to act. Meanwhile, in this study, compared with the Han nationality patients with PTSD, the LI nationality patients with PTSD had higher ETI score, total PTSD score and TMT time, but lower IQ of WAIS-RC comprehension and operation. It has been proved that the TMT B duration of PTSD patients is longer, indicating that the TMT duration of Li and Han people is longer. Besides, WCST showed execution defects, and its errors were used to evaluate POWS for PTSD, with more persistent WCST errors observed in severe cases [22] [29]. Interestingly, according to our study, more total errors were found in patients with 
PTSD [22]. Studies have shown that lower IQ and pre-traumatic educational achievement play a critical role in post-traumatic stress disorder symptoms. verbal memory, working memory, attention performance, and verbal intelligence were negatively correlated with PTSD symptoms [30]. Our study showed that 5-HTTIPR (VNTR) was divided into SS, SL and LL genotypes, while BDNF (rs6265) had multiple homozygous genotypes AA, multiple heterozygous genotypes GA and WT homozygous genotypes GG. The genotypic distribution of 5-HTTIPR (VNTR) and rs6265 in BDNF of Han and Li population was consistent with Hardy-Weinberg equilibrium, suggesting that 5-HTTlPR (VNTR) and rs6265 could be used insubsequent experiments. The SS genotype and S allele may increase the risk of PTSD. Previous studies have shown that 5-HTTLPR (VNTR) genotype has three bialleles, namely SS, SL and LL, and S homozygous in PTSD is higher than L homozygous and heterozygous [9] [31], which is consistent with the results of this study, subjects from the Detroit Community Health Study had genotypes consistent with hardy-Weinberg equilibrium, and the SL genotype was found to be the most common of the three 5-HTTLPR bialleles [4]. Our study showed that SS and S allele in 5-HTTLPR (VNTR) increased the risk of PTSD in Hainan Han population, while AA and A allele in BDNF (rs6265) increased the risk of PTSD in Hainan Han and Li population. As a 43 bp deletion, 5-HTTLPR (VNTR) polymorphism has two alternative genetic variants, including the $S$ allele and the $\mathrm{L}$ allele [32]. Interestingly, the $\mathrm{S}$ allele is a risk factor for PTSD in many traumatic exposures, and the SS genotype also appears to be a specific risk factor for PTSD [4]. At the same time, studies have confirmed that SS genotype has higher risk factors for PTSD in the case of high trauma exposure [33], which is consistent with the results of current studies, patients carrying the S allele of 5-HTTLPR had significantly worse PTSD symptoms than patients carrying the homozygous L allele [25]. Similarly, a higher incidence of PTSD was found compared with L homozygous and heterozygous, and the addition of the S allele of 5-HTTLPR 9 contributed to the severity of PTSD, subjects with the S allele of 5-HTTlPR showed a higher risk of PTSD symptoms than subjects with the homozygous L allele [25]. Our study showed that the $S$ allele and SS genotype of 5-HTTLPR (VNTR) also increased the risk of PTSD in Li and Han populations in Hainan. Previous studies have shown that BDNF (rs6265) polymorphism is thought to interfere with activity-dependent BDNF secretion, thereby increasing synaptic strength, possibly as a means of regulating specific synaptic connections [15] [34] [35] [36]. Compared with GG genotype of BDNF (rs6265), subjects with rs6265 genotype (AG+AA) had an increased risk of PTSD and decreased BDNF expression [16] [37].

\section{Conclusion}

In this study, SS genotype/S allele of 5-HTTLPR (VNTR) and AA genotype/A allele of BDNF (rs6265) were significantly associated with the risk of PTSD in Li and Han Chinese population, and were the main risk factors for PTSD in Li and 
Han Chinese population. In this study, we found that the ETI score, TOTAL PTSD score and TMT time were higher in the Han nationality and Li nationality in Hainan province, but their comprehension, operation IQ and block mode were lower. Additionally, the SS genotype/S-allele in 5-HTTLPR (VNTR) and the AA genotype/A-allele in BDNF (rs6265) increased the risk of PTSD. However, the study had some limitations. First, the polymorphism of 5-HTTLPR (VNTR) and BDNF (rs6265) genes may be affected by population differences. Therefore, this study is more meaningful for a larger sample, population-based differences, particularly in the degree of linkage imbalance between the tested polymorphism and other potential variants in the gene, may have contributed to this inconsistent finding. However, these findings open up a new avenue for research that may provide insights into the relationship between genetic polymorphisms and PTSD.

\section{Fund Source}

National Natural Science Foundation of China (Grant No. 81760255).

\section{Conflicts of Interest}

The authors declare no conflicts of interest regarding the publication of this paper.

\section{References}

[1] Roth, T.L., Zoladz, P.R., Sweatt, J.D. and Diamond, D.M. (2011) Epigenetic Modification of Hippocampal Bdnf DNA in Adult Rats in an Animal Model of PostTraumatic Stress disorder. Journal of Psychiatric Research, 45, 919-926. https://doi.org/10.1016/j.jpsychires.2011.01.013

[2] Chapman, C., Mills, K., Slade, T., McFarlane, A.C., Bryant, R.A., Creamer, M., et al. (2012) Remission from Post-Traumatic Stress Disorder in the General Population. Psychological Medicine, 42, 1695-1703. https://doi.org/10.1017/S0033291711002856

[3] Robinson, B.L. and Shergill, S.S. (2011) Imaging in Posttraumatic Stress Disorder. Current Opinion in Psychiatry, 24, 29-33. https://doi.org/10.1097/YCO.0b013e3283413519

[4] Walsh, K., Uddin, M., Soliven, R., Wildman, D.E. and Bradley, B. (2014) Associations between the SS Variant of 5-HTTLPR and PTSD among Adults with Histories of Childhood Emotional Abuse: Results from Two African American Independent Samples. Journal of Affective Disorders, 161, 91-96. https://doi.org/10.1016/j.jad.2014.02.043

[5] Andero, R., Brothers, S.P., Jovanovic, T., Chen, Y.T., Salah-Uddin, H., Cameron, M., et al. (2013) Amygdala-Dependent Fear Is Regulated by Oprl1 in Mice and Humans with PTSD. Science Translational Medicine, 5, $188 \mathrm{ra} 173$. https://doi.org/10.1126/scitranslmed.3005656

[6] Banerjee, S.B., Morrison, F.G. and Ressler, K.J. (2017) Genetic Approaches for the Study of PTSD: Advances and Challenges. Neuroscience Letters, 649, 139-146. https://doi.org/10.1016/j.neulet.2017.02.058

[7] Forresi, B., Caffo, E. and Battaglia, M. (2015) Gene Environment Interplays: Why PTSD Makes a Good Case for Gene-Environment Interaction Studies and How 
Adding a Developmental Approach Can Help. In: Martin, C., Preedy, V. and Patel, V., Eds., Comprehensive Guide to Post-Traumatic Stress Disorder, Springer, Cham, 1-13. https://doi.org/10.1007/978-3-319-08613-2_84-1

[8] Frielingsdorf, H., Bath, K.G., Soliman, F., DiFede, J., Casey, B.J. and Lee, F.S. (2010) Variant Brain-Derived Neurotrophic Factor Val66Met Endophenotypes: Implications for Posttraumatic Stress Disorder. Annals of the New York Academy of Sciences, 1208, 150-157. https://doi.org/10.1111/j.1749-6632.2010.05722.x

[9] Liu, Y., Garrett, M.E., Dennis, M.F., Green, K.T., Ashley-Koch, A.E., VA Mid-Atlantic MIRECC Registry Workgroup, et al. (2015) An Examination of the Association between 5-HTTLPR, Combat Exposure, and PTSD Diagnosis among U.S. Veterans. PLoS ONE, 10, Article ID: e0119998.

https://doi.org/10.1371/journal.pone.0119998

[10] Porcelli, S., Fabbri, C. and Serretti, A. (2012) Meta-Analysis of Serotonin Transporter Gene Promoter Polymorphism (5-HTTLPR) Association with Antidepressant efficacy. European Neuropsychopharmacology, 22, 239-258. https://doi.org/10.1016/j.euroneuro.2011.10.003

[11] Xu, G.Z., Liu, Y., Zhang, Y., Yu, J. and Diao, B. (2015) Correlation between VEGFR2 rs2071559 Polymorphism and Glioma Risk among Chinese Population. International Journal of Clinical and Experimental Medicine, 8, 16724-16728.

[12] Telch, M.J., Beevers, C.G., Rosenfield, D., Lee, H., Reijntjes, A., Ferrell, R.E., et al. (2015) 5-HTTLPR Genotype Potentiates the Effects of War Zone Stressors on the Emergence of PTSD, Depressive and Anxiety Symptoms in Soldiers Deployed to Iraq. World Psychiatry, 14, 198-206. https://doi.org/10.1002/wps.20215

[13] Zhang, K., Xu, Q., Xu, Y., Yang, H., Luo, J., Sun, Y., et al. (2009) The combined Effects of the 5-HTTLPR and 5-HTR1A Genes Modulates the Relationship between Negative Life Events and Major Depressive Disorder in a Chinese Population. Journal of Affective Disorders, 114, 224-231. https://doi.org/10.1016/j.jad.2008.07.012

[14] Tan, J.M., Wu, J., Shi, J.G., Shi, G.D., Liu, Y.L., Liu, X.H., et al. (2013) Brain-Derived Neurotrophic Factor Is Up-Regulated in Severe Acute Cauda Equina Syndrome Dog Model. International Journal of Clinical and Experimental Medicine, 6, 431-437.

[15] Andero, R. and Ressler, K.J. (2012) Fear Extinction and BDNF: Translating Animal Models of PTSD to the Clinic. Genes, Brain and Behavior, 11, 503-512.

https://doi.org/10.1111/j.1601-183X.2012.00801.X

[16] Green, C.R., Corsi-Travali, S. and Neumeister, A. (2013) The Role of BDNF-TrkB Signaling in the Pathogenesis of PTSD. Journal of Depression and Anxiety, 2013, Sup. 4. https://doi.org/10.4172/2167-1044

[17] Tsai, S.J., Hong, C.J., Liu, H.C., Liu, T.-Y. and Liou, Y.-J. (2006) The Brain-Derived Neurotrophic Factor Gene as a Possible Susceptibility Candidate for Alzheimer's Disease in a Chinese population. Dementia and Geriatric Cognitive Disorders, 21, 139-143. https://doi.org/10.1159/000090673

[18] Chen, T., Guo, M., Gao, Y., Chen, F., Guo, J., Liu, T., et al. (2014) A Comparative Study on the Levels of Serum Cytokines and Cortisol among Post-Traumatic Stress Disorder Patients of Li and Han Ethnicities in Hainan. Chinese Medical Journal, 127, 2771-2774.

[19] Kilpatrick, D.G. (2013) The DSM-5 Got PTSD Right: Comment on Friedman (2013) Journal of Traumatic Stress, 26, 563-566. https://doi.org/10.1002/jts.21844

[20] Kilpatrick, D.G., Resnick, H.S., Milanak, M.E., Miller, M.W., Keyes, K.M. and Friedman, M.J. (2013) National Estimates of Exposure to Traumatic Events and PTSD 
Prevalence Using DSM-IV and DSM-5 Criteria. Journal of Traumatic Stress, 26, 537-547. https://doi.org/10.1002/jts.21848

[21] Blake, D.D., Weathers, F.W., Nagy, L.M., Kaloupek, D.G., Gusman, F.D., Charney, D.S., et al. (1995) The Development of a Clinician-Administered PTSD Scale. Journal of Traumatic Stress, 8, 75-90. https://doi.org/10.1002/jts.2490080106

[22] Zhu, D., Dong, Y., Li, H., Zhang, A. and Liu, Y. (2014) A Study on the Correlation between Coping Style of Traumatic Events and Mental Health of Medical Staff in Earthquake-Stricken Areas. Journal of Clinical Psychosomatic Diseases, 20, 70-75.

[23] Vinod, G. and Jordan, G. (1995) Are the Fmntal L Lobes Implicated In "Planning" Function? Interpreting Data from the Tower of Hanoi. Neuropsyehologia, 33, 623642. https://doi.org/10.1016/0028-3932(95)90866-P

[24] Schmidtke, K., Manner, H., Kaufmann, R. and Schmolck, H. (2002) Cognitive Procedural Learning In patients with Fronto-Striatal Lesions. Learning \& Memory, 9 , 419-429. https://doi.org/10.1101/lm.47202

[25] Kanagaratnam, P. and Asbjornsen, A.E. (2007) Executive Deficits in Chronic PTSD Related to Political Violence. Journal of Anxiety Disorders, 21, 510-525.

https://doi.org/10.1016/j.janxdis.2006.06.008

[26] Su, Y.A., Wu, J., Zhang, L., Zhang, Q., Su, D.M., He, P., et al. (2008) Dysregulated Mitochondrial Genes and Networks with Drug Targets in Postmortem Brain of Patients with Posttraumatic Stress Disorder (PTSD) Revealed by Human Mitochondria-Focused cDNA Microarrays. International Journal of Biological Sciences, 4, 223-235. https://doi.org/10.7150/ijbs.4.223

[27] Chen, Z.J., Zhao, H., He, L., Shi, Y., Qin, Y., Shi, Y., et al. (2011) Genome-Wide Association Study Identifies Susceptibility Loci for Polycystic Ovary Syndrome on Chromosome 2p16.3, 2p21 and 9q33.3. Nature Genetics, 43, 55-59. https://doi.org/10.1038/ng.732

[28] Young, K.A., Morissette, S.B., Jamroz, R., Meyer, E.C., Stanford, M.S., Wan, L., et al. (2017) 5-HTTLPR and DISC1 Risk Genotypes for Elevated PTSD Symptoms in US Military Veterans. World Psychiatry, 16, 109-110. https://doi.org/10.1002/wps.20359

[29] Di Gangi, J.A., Kujawa, A., Aase, D.M., Babione, J.M., Schroth, C., Levy, D.M., et al. (2017) Affective and Cognitive Correlates of PTSD: Electrocortical Processing of Threat and Perseverative Errors on the WCST in Combat-Related PTSD. Progress in Neuro-Psychopharmacology and Biological Psychiatry, 75, 63-69. https://doi.org/10.1016/j.pnpbp.2017.01.004

[30] Aupperle, R.L., Melrose, A.J., Stein, M.B. and Paulus, M.P. (2012) Executive Function and PTSD: Disengaging from Trauma. Neuropharmacology, 62, 686-694. https://doi.org/10.1016/j.neuropharm.2011.02.008

[31] Braithwaite, E.C., Ramchandani, P.G., O’Connor, T.G., van Ijzendoorn, M.H., Bakermans-Kranenburg, M.J., Glover, V., et al. (2013) No Moderating effect of 5HTTLPR on Associations between Antenatal Anxiety and Infant Behavior. Journal of the American Academy of Child and Adolescent Psychiatry, 52, 519-526. https://doi.org/10.1016/j.jaac.2013.02.010

[32] Plieger, T., Montag, C., Felten, A. and Reuter, M. (2014) The Serotonin Transporter Polymorphism (5-HTTLPR) and Personality: Response Style as a New Endophenotype for Anxiety. International Journal of Neuropsychopharmacology, 17, 851-858. https://doi.org/10.1017/S1461145713001776

[33] Hirohashi, S., Clausen, H., Nudelman, E., Inoue, H., Shimosato, Y. and Hakomori, S. (1986) A Human Monoclonal Antibody Directed to Blood Group I Antigen: He- 
terohybridoma between Human Lymphocytes from Regional Lymph Nodes of a Lung Cancer Patient and Mouse Myeloma. Journal of Immunology, 136, 4163-4168.

[34] Karnik, M.S., Wang, L., Barch, D.M., Morris, J.C. and Csernansky, J.G. (2010) BDNF Polymorphism rs6265 and Hippocampal Structure and Memory Performance in Healthy Control Subjects. Psychiatry Research, 178, 425-429.

https://doi.org/10.1016/j.psychres.2009.09.008

[35] Zhang, L., Benedek, D.M., Fullerton, C.S., Forsten, R.D., Naifeh, J.A., Li, X.X., et al. (2014) PTSD Risk Is Associated with BDNF Val66Met and BDNF Overexpression. Molecular Psychiatry, 19, 8-10. https://doi.org/10.1038/mp.2012.180

[36] Zivadinov, R., Weinstock-Guttman, B., Benedict, R., Tamaño-Blanco, M., Hussein, S., Abdelrahman, N., et al. (2007) Preservation of Gray Matter Volume in Multiple Sclerosis Patients with the Met Alele of the rs6265 (Val66Met) SNP of Brain-Derived Neurotrophic Factor. Human Molecular Genetics, 16, 2659-2668.

https://doi.org/10.1093/hmg/ddm189

[37] Zakharyan, R. and Boyajyan, A. (2014) Brain-Derived Neurotrophic Factor Blood Levels Are Decreased in Schizophrenia Patients and Associate with rs6265 Genotypes. Clinical Biochemistry, 47, 1052-1055.

https://doi.org/10.1016/j.clinbiochem.2014.03.021 